\title{
Reply to the letter by Takeshi Yamada et al. concerning "Is serum HER2 ECD a predictive biomarker for response to trastuzumab in advanced gastric cancer?"
}

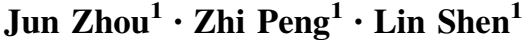

Received: 24 January 2016/Accepted: 9 February 2016/Published online: 20 February 2016

(C) Japanese Society of Gastroenterology 2016

To the editor:

We thank Yamada et al. for their constructive comments entitled "Is serum HER2 ECD a predictive biomarker for response to trastuzumab in advanced gastric cancer?" related to our article [1].

In our study, an objective response rate (ORR) was not shown by subgroups because most patients ( 48 of 65 ) were treated with first-line chemotherapy. In those 48 patients, those with higher baseline serum human epidermal growth factor receptor 2 (HER2) extracellular domain (ECD) levels $(15.0 \mathrm{ng} / \mathrm{mL}$ or more and $10.65 \mathrm{ng} / \mathrm{mL}$ or more) had a better ORR $(89.5 \%$ vs $52.0 \%, P=0.01 ; 81.3 \%$ vs $33.3 \%, P=0.004$ ) than those with normal baseline serum HER2 ECD levels (less than $15.0 \mathrm{ng} / \mathrm{mL}$ and less than $10.65 \mathrm{ng} / \mathrm{mL}$ ). Because of the limited number of patients, in the 14 patients treated with second-line trastuzumabbased chemotherapy, a higher baseline serum HER2 ECD level was still associated with better ORR although not to a statistically significant extent.

We found a high baseline serum HER2 ECD level and a decline of serum HER2 ECD level during chemotherapy significantly correlated with the response to chemotherapy, which is consistent with the findings reported in another

This authors' reply refers to the letter to the editor at doi:10.1007/ s00535-016-1184-2.

Lin Shen

lin100@medmail.com.cn

Zhi Peng

zhipeng3@hotmail.com

1 Department of Gastrointestinal Oncology, Key Laboratory of Carcinogenesis and Translational Research (Ministry of Education), Peking University Cancer Hospital and Institute, FuCheng Road 52, HaiDian District, 100142 Beijing, China article concerning gastric cancer [2]. In breast cancer, there are indeed contrary results concerning the predictive value of serum HER2 ECD levels in HER2-positive breast cancer [3]. However, the mechanism is still unclear.

In our observational study we prospectively collected consecutive HER2-positive advanced gastric cancer patients treated with trastuzumab-based chemotherapy. We could not exclude the potential bias due to the heterogeneity of the patients. As we discussed in the "Discussion" section, further well-designed prospective studies with a larger sample size need to be performed to validate and strengthen our conclusion.

Compliance with ethical standards

Conflict of interest The authors declare that they have no conflict of interest.

\section{References}

1. Zhou J, Peng Z, Liu Y, et al. Predictive value of serum HER2 ECD in patients with HER2-positive advanced gastric cancer treated with trastuzumab plus chemotherapy. J Gastroenterol. 2015;50:955-61.

2. Oyama K, Fushida S, Tsukada T, et al. Evaluation of serum HER2ECD levels in patients with gastric cancer. J Gastroenterol. 2015;50:41-5.

3. Lam L, McAndrew N, Yee M, et al. Challenges in the clinical utility of the serum test for HER2 ECD. Biochim Biophys Acta. 2012;1826:199-208. 